\title{
Large-Vocabulary Speaker-Independent Continuous Spech Recognition with Semi-Continuous Hidden Markov Models
}

\author{
X.D. Huang*, H.W. Hon, and K.F. Lee \\ School of Computer Science \\ Carnegie Mellon University \\ Pittsburgh, PA 15213
}

USA

\begin{abstract}
A semi-continuous hidden Markov model based on the multiple vector quantization codebooks is used here for large-vocabulary spesker-independent continuous speech recognition in the techniques employed here. the semi-continuous output proba. bility density function for each codebook is represented by a combination of the correxponding discrele output probubililies of the hidden Mlarkor model and the continuous Gausian den. sity functions of each individual codebook. Parameters of vec. tor quantization codebook and hidden Markov model are mutu. dly optimized to achieve an optimal model codebook combina. tion under a unified probabilistic framework Another advan. tages of this approach is the enhanced rubustness of the sem: continuous output probability by the combination of multuple codewords and muluple codebooks For a 1000-word speaker. independent continuous speech recognition using a word-pair grammar. the recognition error rale of the semi-continuous hid. den Markor model was reduced by more than $29 \%$ and $41 \%$ in comparison to the discrete and continuous mixture hidden Mar. kov model respectively
\end{abstract}

\section{INTRODUCTION}

In the discrete bidden Markov model (HMM), vector quantization $1 Q$. produces the closet codebword from the codebook for each acoustic observation. This mepping from continuous ecoustic space to quantized discrete space may cause serious quantization errors for subsequent hidden Markor modeling. To reduce $\vee Q$ eprors. various smootbing techniques have been proposed for $V Q$ and subsequent bidden Markor modeling [9.12). A distinctive lechnique is multiple VQ codebook bidden Markor modeling. which has beea shown to offer improved speech recognition eccu. racy $(5,12)$. In the multiple VQ codebook approuch, VQ dietortion can be significantly minimized by partitioning the parameters into separale codebooks. Another disadvantage of the discrete HMM is that the VQ codebook and the discrete HMM are separately modeled. which may not be an optimal combination for pattern classification (8). The discrete HMM uses the discrete output probability distributions to model various acoustic events. which are inberently superior to the coatiouous mixture HMM with mixture of small aumber of probability deasity functions since the discrete distributions could model events with any shapes pro. vided enough training data exist.

On the other hand. the continuous mixture HMM models the acoustic observation directly using estimated continuous probabil. ity deasity functions without VQ. and has been shown to improve the recognition accuracy in comparinon 10 the discrete HMM ! 15! For speaker-independent speech recognition, mixture of a large number of probability density functions $(14.16)$ or a large number of states in sungle-mixture case [ 4$]$ are generally required to model characteristucs of different speakers. However, mixture of a large number of probability density functions will considerably increase not only the computational complexity, but also the number of tree parameters that can be reliablely estimated. In addition. the continuous mixture HMM has to be used with care as continuous

- and Univernily of Edinburgh, 80 South Bridge. Edinburgh EHI IHN, UK probability density functions make more assumption than the discrete HMM, especially when the diagonal covariance Gaussian probability densily is used for simplicity [15]. To obtain a better recognition accuracy. acoustic parameters must be well chosen according to the assumption of the continuous probability density functions used

The semi.continuous hidden Markov model 'SCHMM) has been proposed to extrnd the discrete HMAl by replacing discrete output probability distributions with combination of the original discrete output probabilaty distributions and continuous probabil. 1 ty density functions of a Gaussian codebook (6). In the SCHMM. each VQ codeword is regarded as a Caussian probability denaity Intuluvely, from the discrete HMIM point of view, the SCHMM tries to smooth the discrete output probabilities with multiple codeword candidates in $V Q$ procedure. From the continuous mix. ture HMM point of view, the SCHMM ties all the concinuous out. put probability densities across each individual HMM to form a shared Gaussian codebook. i.e. a mixture of Gaussian probability densilies. With the SCH.ITM. the codebook and HMM can be jointly reestumated to achieve an optimal codebook model combi. nation in sense of maximum likelihood criterion. Such a tying can also substantially reduce the number of free parameters and com. putational complexity in comparison to the continuous mixture HMM. while mairtain reasonablelv modeling power of a mixture of a large number of probability deasity functions. The SCHMIM has shown to offer improved recognition accuracy in several seecb recognition experiments $(6.8,14.2)$.

In this study. the SCHMM is applied to Sphinx, a speaker independent continuous speech recognition system. Sphinx uses multiple $Q$ codebooks for each acoustic observation [12]. To apply the SCHMM to Sphinx. the SCHMM algorthm must be modified to sccommodate multiplo codebooks and multiple codewords combination. For the SCHMM re-estimation algoritbm. the modified unifed re-estimation algorithm for multiple $V Q$ code. books and bidden Markov models are proposed in this paper. The applicability of the SCHMM to speaker-independent continuous apeech is explored based on 200 generalized triphone models [12]. in the 1000-word speaker-independent continuous speech recogni. tion lask using word-pair grammar. the error rate was reduced by more than 29\% and $41 \%$ in comparison to the corresponding discrete $H M M$ and concinuous mixture HMM respectively

\section{SEMI.CONTINUOUS HIDDEN MARKOV MODELS}

\subsection{Discrete H.H.Ms and Continuous HMMs}

Aa Nistate Markov chain with state transition matrix $A=\left[n_{1}\right]$. $i j=1.2$.... N. where $a_{1}$ denotes the transition probability from state $i$ to state $j$; and discrete output probubility distribution. $6.10,1$ or continuous output probability density function $0,1 \times 1$ associated with each state $f$ of the unobservable Markov chain is considered here. Here $O$, represents discrele observation symbols tusually. VQ indicesi, and $x$ represents continuous observations (usually speech frame vectorsi of K.dimensional random vectors.

With the discrete HMM. there are $L$ discrete output symbols from - Lievel VQ. and the output probability is modeled with discrete probability distributions of these discrete symbols. Let $O$ be the observed sequence, $O=O_{4} O_{n_{2}} \cdots O_{4}$, observed over $T$ samples. Here $O_{4}$, denotes the $Y Q$ codeword $k_{1}$ observed at time $i$. The 
observation probability of such an observed sequence. Pr $O \mid \lambda_{1}$, can be expressed as:

$$
\operatorname{Pr}(0 \mid \lambda)=\sum_{s} \pi \prod_{1=1}^{r} a_{1-1}, b_{1,}\left(O_{1,1}\right.
$$

where $S$ is a purticular state sequence. $S \in\left(s_{n} s_{1}, \ldots, s, s_{,} \in \| 1\right.$. 2. . N!. and the summation is taken over all of the possible state sequences. $S$. of the given model $\lambda$. which is represented by $i \pi, A$, $B_{1}$, where $\pi$ is the initial state prohability vector. $A$ is the state trinsition matrix. and $B$ is the output probability distribution matrix. In the discrete HMM. classification of $O_{h}$ from $x_{1}$ in the $V Q$ may not be accurate.

If the observation to be decoded is not vector quantized. then the prohability denasty function, $f X \mid \lambda$. of producing an observation of continuous vector sequences given the model $\lambda$, would be computed. instead of the probability of generating a discrete ohserva. tion aymbol. PriOl $\lambda$. Here $X$ is a sequence of continuous acous. lis vectors $x, X=x_{1} x_{2} \cdots x_{T}$. The principal advantage of using the continuous HMM is the ability to directly model speech parameters without involving VQ. However, the continuous HMIM requires considerably longer training and recognition times. especially when a mixture of several Gaussian probability density components is used. In the continuous Gaussian IM-componenti mixture HMM 1111 . the output probability density of state $J . b,(x)$. can be represented as

$$
b_{j}(\boldsymbol{x})=\sum_{i=1}^{M} c_{, k} N\left(\boldsymbol{x}, \mu, \boldsymbol{\Sigma}, \Sigma^{\prime}\right.
$$

where $\rightarrow(x, \mu, \Sigma$ ' denotes a multi-dimensional Gaussian density function of mean vector $\mu$ and covariance matrix $\sum$. and $c_{j}$, is a weighting coefficient for the kth Gaussian component With sucb a mixture, any arbitrary distribution can be approximately modeled, provided the mixture is large enough.

\subsection{Semı.Continuous Hidden Markou Models}

In the discrete HMM. the discrete probubility diatrihutiona are sufficienity powerful to model any random events with a reason. able number of parameters. The major prohlem with the discrete output prohability is that the VQ operation partitions the acoustic space into aeparate regions according to some distortion measure. Thin introduces errors as the partition operations may destroy the original signal structure An improvement is to model the VQ codebook as a family of Gaussian density functions such that the distributions are overlaped, rather than disjointed. Each codeword of the corehook can then be represented by one of the Gaussian probability density functiona and may be used together with oth. ers to model the acoustic event. The use of a parametric family of finite mixture densities a mixture density $V Q$ can then be closely combined with the HMM methodology. From the continuous mixture HMM point of view. the output probability in the con. tinuous mixture HMM is shared among the Gaussian probability density functions of the $V Q$. This can reduce the number of free parameters to be estimated as well as the computational complex. ity. From the discrete HMM point of view, the partition of the $V Q$ is unnecessary, and is replaced by the mixture density model. ing with overlap. which can effectively minimize the $V^{\prime} Q$ errors. The procedure. known as the EM algorithm [3]. is a specialization. to the mixture density context, of general algorithm for obtain. ing maximum likelihood estimates. This has been defined earlier by Baum [1] in similar way and has been widely used in HMM. based speech recognition methods. Thus, the VQ problems and HMM modeling problems can be unified under the same proba. bilistic framework to obcain an optimized VQ'HMM combination, whicb forms the foundation of the SCHMM.

Provided that each codeword of the VQ codebook is represented by a Gaussian density function, for a given state $s_{t}$ of HMM, the probability density function that $s$, produces a vector $x$ can the be written as:

$$
b_{s_{t}}(x)=f\left(x \mid s_{t}\right)=\sum_{l=1}^{L} f\left(x \mid O_{l_{t}}, s_{t}\right) \operatorname{Pr}\left(O_{j_{t}} \mid s_{t}\right)
$$

where $L$ denotes the VQ codebook level. For the sake of simplicity, the output probability density function conditioned on the codewords can be assumed to be independent of the Markov states $s_{f},(3)$ can then be written as

$$
f\left(x \mid s_{l}\right)=\sum_{l=1}^{L} f\left(x\left|O_{d_{1}}\right| \operatorname{Pr}\left|O_{d_{1}}\right| s_{1}\left|=\sum_{l=1}^{L} f\right| x\left|O_{l_{1}}\right| b_{l_{1}} \mid O_{\lambda_{1}}{ }^{\prime}\right.
$$

This equation is the key to the semi-continuous hidden Markov

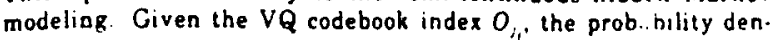
sity function $f\left(x \mid O_{,}\right.$, can be estimated with the EM algorithm $\{17\}$. or maximum likelihood clustering. It can also be obtained from the HMM parameter estimation directly as explained later. Cising 14) to represent the semi.continuous oulput probability density. it is possible to combine the codebook distortion characteristics with the parameters of the discrete HMM under a unified probabilistic framework. Here. each discrete output probability is weighted by the continuous conditional Gaussian probability density function derived from VQ if these continuous VQ density functions are considered as the continuous output probahility density function in the continuous mixture HMM. this also resembles the $L$. mixture continuous $H M M$ with all the continuous output probahil. ity density functions shared with each other in the VQ codebook Here the discrete output probabtlity in state $1, b, 1,1$, , becomes the weightung coefficients for the mixture components.

In implementation of the SCHMM [8], Eq. 141 can be replaced by finding $M$ most significant values of $f(x \mid O$ ) ( with $M$ be one to six. the algorithm converges well in practicel over all possible codebook indices 0 , which can be easily oblained in the VQ pro. cedure. This can significantly reduce the amount of computational load for subsequent output probability computation since $M$ is of lower order than $L$ Experimental results show this to perform well in speech recognition $[8]$, and result in an L-mixture continuous HMM with a computational complexity significantly lower than the continuous mixture HMM

\subsection{Re-estimaiton formulas for the SCHM.M}

If the $b_{1}\left(O_{1}\right)$ are considered as the weighting coefficients of different mixture output probability density functions in the continuous mixture iisili. the recstimation algorthm for the weighting coefficients can be extended to re-estimate $h_{1} \cdot O_{\lambda_{t}}{ }^{\prime}$ of the SCHMM [11]. The re-estimation formulations can be more readily computed by defining a forward partial probability, $a_{t}(i)$, and a backward partial probability, $\beta_{1}(i)$ for any time $t$ and state $i$ as:

$$
\begin{aligned}
& a_{t}(i)=\operatorname{Pr}\left(x_{1}, x_{2}, \cdots x_{t} s_{t}=i \mid \lambda\right) \\
& \beta_{1}(i)=\operatorname{Pr}\left(x_{t+1}, x_{1}+2, \cdots x_{T} \mid s_{t}=i, \lambda\right)
\end{aligned}
$$

The intermediate probabilities, $x_{i}^{\prime}(j, k), Y_{i}(i v), Y_{i}(i), \zeta(i J)$. and $\zeta(j)$ can be defined as follows for efficient re-estimation of the model parameters:

$$
\begin{aligned}
& X_{1}(i j, k)=\operatorname{Pr}\left(s_{1}=i, s_{1+1}=j, O_{k_{1}} \mid X, \lambda\right) \\
& y_{1}(i j)=\operatorname{Pr}\left(s_{1}=i, s_{t+1}=j \mid X, \lambda\right) \\
& \gamma_{i}(i)=\operatorname{Pr}\left(s_{t}=i \mid X, \lambda_{1}\right. \\
& \zeta_{1}(i, k)=\operatorname{Pr}\left(s_{t}=i, O_{4} \mid X, \lambda\right) \\
& \zeta_{t}(k)=\operatorname{Pr}\left(O_{h} \mid X, \lambda\right)
\end{aligned}
$$

Al! these intermediate probabilities can be represented by $x,{ }^{1}$, Using Eq. 15 ' and 16 , the re-estimation equations for $\pi_{1}, a_{\ldots}$, and $b,(0$,$) can be written as:$

$$
\begin{gathered}
\pi_{1}=\gamma_{1}(i), \quad 1 \leq i \leq N, \\
\vec{a}_{1 j}=\frac{\sum_{i=1}^{T} \gamma_{1}(i j)}{\sum_{i=1}^{T} \gamma_{i}(i)}, \quad 1 \leq i, j \leq N_{i} \\
\bar{b}_{1}(O)=\frac{\sum_{i=1}^{T} \zeta_{i}((j)}{\sum_{i=1}^{T} \gamma_{!}(i)}, \quad 1 \leq i \leq N_{i} \quad 1 \leq j \leq L .
\end{gathered}
$$

The means and covariances of the Gaussian probability densıty functions can alco be reestimated to update the $\mathrm{VQ}$ codebook separately with $E_{q} 151$ and $(6)$. The feedback from the HMM esti- 
mation results to the VQ codebouk implies that the VQ codebnok is optimized based on the HMM likelihood maximization rather than minimizing the total distortion errors from the set of train. ing data. Although re-estimation of means and covariances of different models will involve inter-dependencies, the different den. sity functions which are re-estimated are strongly correlated. To re-estimate the parameters of the $V Q$ codebook, i.e. the means. $\mu_{j}$. and covariance matrices. $\Sigma$, of the codebook index $j$. it is not difficult to extend the continuous mixture HMM re-estimation algorithm with modified $Q$ function. In general, it can be written as:

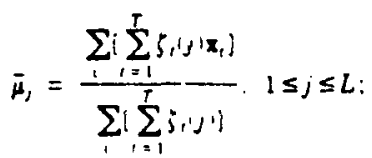

and

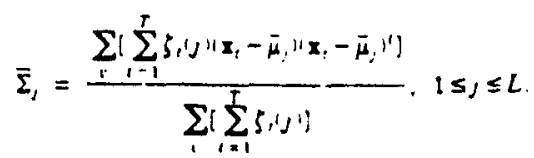

where 1 denotes the HMM used: and expressions in ! I are vari. uhles of model ( . In F.a 110 ) and 111 , the re eatimatinn for the means and covariance matrices in the output probability density function of the SCHMM are tied up with all the HMM models. which is similar to the approach with tied transition probability inside the model [10]. From F.q 110 , and 111 , It can he observed that they are merely a special form of E.I algorithm for the parameter estimation of mixture density functions? $1:$. which are closely welded into the HMM re-estimation equations

When multiple codebooks are used. each codebook represents a set of different speech parameters. One way to combine these multiple output observations is to sssume that they are independent. and the output probability is computed as the product of the out. put probability of each codebook. It has been shown that perfor. mance using multiple codebook can be substationally improved [13]. In the semi-continuous HMM. the semi-continuous output probability of multiple codebooks can also be computed as the pro. duct of the semi-continuous output probability for each codebook us Eq 141. which consists of L-mixture continuous density fun: tions. In other word, the semi-continuous output probability could be modified as.

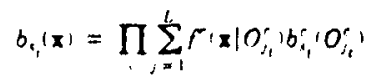

wherc $r$ denotes the codebonk used. The reestimation algorithm for the multiple codebook based HMM could be extended if Eq. 16 a 1 is computed for each codeword of each codebook $c$ with the combination of the rest codebook probability [ 7 ].

\section{EXPERIMENTAL EVALUATION}

\subsection{Analysis Conditions}

For botb training and evaluation. the standard Sphinx frontend consists of 12th order bilinear transformed LPC cepstrum (12). The complete database consists of $\$ 3.58$ training sentences from 105 speakers (june-train and 300 test sentences from 12 speakers.

The vocabulary of the Resource Management database is 991 words. There is also an official word.pair recognition grammar. wbich is just a list of allowable word pairs without probabilities for the purpose of reducing the recognition perplexity to about 60 .

\subsection{Experimental Results Using Bilinear Transformed Cepstrum}

Discrete HMMs and continuous mixture HMMs based on 200 gen. eralized triphones are first experimented as benchmarks. The discrete HMM is the same as Sphiax except only 200 generalized triphones are used [12].

In the continuous mixture HMM implemented here, the cepstrum. difference cepstrum. normalized energy, and difference energy are packed into one vector. This is similar to the one codebook imple. mentation of the discrete HMM [12]. Each continuous output prohability consista of 4 diagonal liausaian pruhahility denatly function as $\mathrm{Eq}_{\mathrm{q}}$, 2) To obcain seliable initial modela for the con. tinuous mixture HMM. the Viterhi alignment with the discete HMM is used to phonetically segment and label truining speech. These labeled segments are then clustered by using the $k$-means clustering algorithm to obtain initial means and diagonal covari. ances. The forward-backward algorithm is used iteratively for the monophone models, which are then used as initial models for the generalized triphone models. Though contunuous mixture HAM was reported to significantly better the performance of the discrete HMM [15]. for the experiments coaducted bere, it is significantly worse than the discrete HMM. Why is this paradox? One expla. nation is that multiple codebooks are used in the discrete HMM. therefore the VQ errors for the discrete HMM are not so serious here. Another reason may be that the diagonal covariance assumption is not appropriate for the bilinear transformed LPC cepatrum since many coefficients are strongly correlated after the transformation. Indeed, observation of average covariance matrix for the bilinear transformed LPC cepstrum shows that values of off-diagonal components are generally quite large.

For the semi-continuous model, multiple codehooks are used instead of packing different feature parameters into one vector The initial model for the SCHMM comes directly from the discrete $H M M$ with the $V Q$ variance oblained from $k$-mein $n$ clustering for each codeword In compuling the semi-cuntinuous oulput probabil. lly density function. only the $\mathbf{M}+1$. there most alenificant code. words are used for subsequent prucessing. Under the same analysis condition, the percent correct 'correct word percentage' and word accuracy 'percent correct - percent insertionl results of

\begin{tabular}{|c|c|}
\hline \multicolumn{2}{|c|}{$\begin{array}{l}\text { Table } 1 \\
\text { Average recogaition accuracy } \\
\end{array}$} \\
\hline ives & percent correct (uord accuracy) \\
\hline $\begin{array}{c}\text { Discrete HMM } \\
\text { Continuous Wixture HMM } \\
\text { SCHMM - lopl } \\
\text { SCHMM - lopt }\end{array}$ & $\begin{array}{l}89.5 \%(88.0 \%) \\
84.2 \%(81.3 \%) \\
8 \% .2 \%, 84.0 \%) \\
906 \%(89.1 \%) \\
\end{array}$ \\
\hline
\end{tabular}
the discrete HMM. the continuous mixture HMM, and the SCH.MM are shown in Table 1.

From Table 1. it can be observed that the SCHMM with top 4 codewords works better than both the discrete and continuous mixture HMM. The SCHMM with top I codeword works actually worse than the discrete H:MM, which indicates that diagonal Gaussian assumption may be inappropriate here. Though bilinear transformed cepstral coefficients could not be well modeled by the diagonal Gaussian assumption ' which was proven by the poor performance of the continuous mixture HMM and the SCHMM with Gaussian assumption (which was proven by the poor performance of the continuous mirture HMM and the SCHMM with top 1 codeword), the SCHMM with top 4 codewords works modestly better than the discrete HMM. The improvement may primarily come from smoothing effect of the SCHMM, i.e. the robustness of multi. ple codewords and multiple codebooks in the semi-continuous out. put probability representation. albeit 200 generalized triphone models are relatively well trained in comparison to standard Sphinx version [12], where 1000 generalized triphone models are used.

\subsection{Experimental Results Using Less Correlated Data}

If the diagonal Gausaian covariance is used, each dimension in speech vector should be un-correlated. In practice, this can be partially satisfied by using less correlated feature as acoustic observation representation. One way to reduce correlation is principal component projection. In the implementation here, the projection matrix is computed by first pooling together the bilinear transformed cepstrum of the whole training sentences, and then computing the eigenvector of that pooled covariance matrix. Unfortunately. only insignificant improvements are obtained based on such a projection [ 7 ]. This is because the covariance for each codeword is quite different. and such a projection only makes acerage covariance diagonal, which is inadequate 
As hilinear transformed cepstral coefficients could not be well modeled by diagonal Gauscian probuhility density function. exper iments without bilinear transformation are conducted The isth order cepstrum is used here for the SCHMIM because of less corte lated characteristics of the cepatrum With 4358 training sen tences (june-train', test results of 300 sentences (june-test) are listed in Table?

\begin{tabular}{|c|c|}
\hline \multicolumn{2}{|c|}{$\begin{array}{c}\text { Table } 2 \\
\text { Average accurscy of listb order cepstrum }\end{array}$} \\
\hline types & percent correct (unded accurncy) \\
\hline Discrete $\mathrm{HMM}$ & $=\frac{86.3 r(83.8 r 1}{1}$ \\
\hline SCHMM + topl & $866 \%(855 \%)$ \\
\hline SCHMM + Lop2 & $888 \div 1876 r 1$ \\
\hline SCHMM + top4 & ו וז5 \\
\hline SCHMM + top 6 & $896 r: 1786 r i 1$ \\
\hline SCHMM - top & $893 r+982 r+1$ \\
\hline
\end{tabular}

Herc. the recognition accuracy of the SCHMIM is significanlly improved in comparison with the discrete HMM and error reduc. tion is over 29\% Even the SCHMM with top one codeword is used. it is still better than the discrete HMM i85.5\% vs. $8.38 \%$ Lse of multiple codewords topt and top6i in the semi-contunuous output probability density function greatly improves the word accuracy 1 rom $85.5 \%$ to $88.6 \%$. Further increase of codewords used in the semi-continuous output probability density functions shows no improvement on word accuracy. but substantial growth of computational complexity. From Table 2 , it can be seen that the SCHMM with top four codewords is adequate $1885 \%$. In contrast. when bilinear transformad data was used. the error reduction is less than $10 \%$ in comparison to the discrete HMM and the SCHMM with top one codeword is actually slighily wore than the discrete HM.M. This strongly indicates that approprlate feature is very important if continuous probability density function. espe. cially diagonal covariance assumption. is used If assumption is inappropriate, maximum likelihood estimation will only maximize the lirang assumption Although more than $29 \%$ error reduction has been achieved for 18 th order LPC analyais using diagonal covariance assumption. the last results with the discrete HMM tbilinear transformed cepstrum, $883 \mathrm{~m}$ and the SCHMM $118 \mathrm{~h}$ order cepsirum. $A^{\prime} 6^{\prime} ;$, are ahout the game This augrest that bif. inear tranaformation "helpful for recognitiun. but have corre laled cueficients, which in inappropriate to the dingonal (iausalan assumption. It can be expected that with the full covariance SCHMM and bilinear transformed cepstral duta, better recogni. tion accuracy can be obtained

\section{CONCLUSIONS}

Semi-continuous hidden Markov models based on multiple vector quantization codebooks take the advantsges of both the discrete HMM and continuous HMM. With the SCHMM, it is possible to model a mixture of a large number of probability density func. tions with a limited amount of training data and computational complexity. Robustness is enhanced by using multiple codewords and multiple codebooks for the semi-continuous output probability representation. In addition. the $\mathrm{VQ}$ codebook itself can be adjusted together with the HMM parameters in order to obtain the optimum maximum likelithood of the HMM. The applicability of the continuous mixture HMM or the SCHMM relies on appropriately chosen acoustic parameters and assumption of the continuous probability density function. Acoustic features must be well represented if diagonal covariance is applied to the Gaussian probability density function. This is strongly indicated by the experimental results based on the bilinear transformed cepstrum and cepstrum. With bilinear transformation, high frequency com. ponents are compressed in comparison to low frequency components $(2.3)$. Such a transformation converts the linear fre. quency axis into a mel-scale-like one. The discrete HMM can be substantially improved by bilinear transformation However. bil. inear transformation introduces strong correlations. which is inap. propriate for the diagonal Gausalan assumption modeling. Using the cepstrum without bilinear transformation. the diagonal SCHMM can be substantially improved in comparison to the discrete HMM
All experiments conducted here were based on only 2nn general. ized triphones as smoothing can play a more important rule in those less-well-trained models. more improvement can be expected for 1000 generalized triphones iwhere the word accuracy for the discrete HMM is $91 \%$ with bilinear transformed datal in addi. tion. removal of diagonal covariance assumption by use of full covariance can be expected to further improve recognition accuracy [1]. Regarding use of full covariance, the SCHMM has a dis. tinctive advantage Since Gaussian prohubility density functions are tied to the VQ codebook, by chosing $M$ most significant code. words. computational complexily can be several order lower than the conventional continuous mixture $11 M M$ while matntaning the modeling power of large mixture components

Experimental resulta have clearly demonatrated that the SCHMM offers improved recognition accuracy in compurison to hoth the discrete HMM and the continuous mixture HMM in sperikerindependent continuous speech recognition. We conclude that the SCHMM is indeed a powerful technique for modeling nonstationary stochastic processes with multi-modal probabilistic functions of Markov chains.

\section{ACKNORLEDGEMENTS}

We would like to thank Profesoor Mervyn Jack and Profesmor Raj Rertiy for their heip and insight shared in this research

\section{REFERENCES}

(1) Buum, $L \varepsilon$ and et al. "A maximization technique occuring in the ata. tuatical analysis of prohabiluatic functions of Murkov chains". J Ann Math Stut Vol 41. pp \$64.131.1970

21 Bellegarda. I and Sahamon. D. "Tied mixture continuous parameter model. for large vocabulary isolated speech recognation". ICASSP 89. Glangox. Scotlund. 1989

13) Dempater. A P. Laird. N M and Rubin, D B "Muximum.likelihood from incomplete data via the EM algorthm". J. Royal Statist Soc. Ser. B imethodological. Yol 39. pp 1.38.197T

14) Doddington. G R. "Phoneticalty senastive discriminants for improved upeech recornition", IC ASSP 89, Glaagow. Scotland, 1989

(5) Gupta. V N Lennia. M and Mermeistein, $P$ "Integration of Acous. tic Informition in a Liarge Vocabulary Word Recognizer". ICASSP 87, pp. 697.700 .1987

h) Huang. X D wl Jick. MA. "Hidden Murkov modelling of speech hraed on yemi cuntinuuts model", Electronica Letters, Vul. 24. pp 6.7. iyak

17) Huang. X D. Hon. HW aod Lee. K F., Mulliple codebook nemi. contiououn hidden Markov models for speaker-independent continuous speech recognition". CMU Technical Report CMU-CS-89-136. C. Scieace. 1989

(8) Huang, X.D and Jack, M A., "Semi.continuoug hidden Markov models for speech recogaition". Computer Speech and Langugge., Vol. J. 1989

(9) Jelinek. $F$. "Continuous speech recognition by statustical methods", Proceedinge of IEEE. Vol. 64. Pp 532.556. 1976

$110 \mid$ Jeliaek. F. and Mercer. R.L. "Interpolated eatimation of Markov gouree parameters from sparse data". The workshop on pattern recogni. tion in practice. Amaterdam. 1980

IIII Juang. B H. "Maximum.likelihood eatumation for mixture multivari. ate stochastic observations of Markov chain". AT\&T Technical Journal Vol 64, PP 12.35.1249.1985

$112 \mid$ Lee. K F. "Large-vocabulary speaker-independent continunus apeech recognition The SPHINX ssiem". Ph D thess. C Science, CMU 198R [13] Lee. KF. Hon $H W$ and Redr!, $R$. "The SPHINX speech recogn. tion system", IC ASSP R9. Glasgow. Scotland. 1989

(14) Paul, D. "The Lincoln continuous speech recognition syatem recent developmenta und results". DARPA 1989 Feb meeling. 1989

(IS) Rabiner, $L R$ and $e$ al. "Recognition of isolated digits using hidden Markov models with continuous mixture densities". AT\&T Technical Jouraal. Vol 64. pp 1211-1234.1985

[16] Rabiner, L R. Wilpon, J G., and Soong. F.K. "High Pefformance Condected Digit Recognition Using Hidden Markov Modela". ICASSP 88. NY. 1988

[17] Rednes, RA and Walker. H.F., "Mixture densities. muximum likeli. hood and the EM algorithm". SLA.M review. Vol. 26. pp. 195-239. 1984 [18] Shikano. K. "Evaluation of LPC apectrul matching meaquien for phonetic unit recognition". CMU Technical Report CMU.CS.86-108, C Science. 1986 